\title{
Denken und Handeln für unsere Zukunft
}

\begin{abstract}
Um für nachhaltige Entwicklung wirkungsvoll Denken und Handeln hinsichtlich Bewegung, Ernährung, Konsum, Besitz, Energie und Engagement (\#BEKBEE) aus Kap. 1 zu verbinden, kann jeder Tag für Tag bei sich beginnen. Das Dokument mit dem Titel 13 Kilo leichter unter diesem Link https://entfaltungsagentur.files.wordpress.com/2019/08/13_ kilo_leichter_ronald_deckert_2013.pdf bietet einen Erfahrungsbericht, mit dem ich meine ersten Erfahrungen zum Fasten teile. Nun muss sich nicht jede oder jeder mit Fasten anfreunden. Der Erfahrungsbericht kann dazu dienen, mittels eines Beispiels an vielfältigen Erlebnissen rund um eine Veränderung teilzuhaben. Mir persönlich ist Zugang zu Veränderung am liebsten, der bei mir beginnt: Wenn ich ganz bei mir bin, erlebe ich Denken, Fühlen und Handeln sehr direkt und lasse dies auf mich wirken, um diese Erfahrungen dann auch für andere Kontexte wirksam werden zu lassen.
\end{abstract}

Folgendes Zitat von Aoun (2017, S. 87) betont - neben Kreativität, Unternehmergeist und kultureller Agilität - seine in Abschn. 2.2.3 genannte Einordnung zur Fähigkeit des Menschen, Verbindungen zwischen zunächst einmal weit auseinanderliegenden Themenstellungen herzustellen (,far transfer"):

„,...] no computer has yet displayed creativity, entrepreneurialism, or cultural agility. [...] they cannot perform far transfer well, at least not in the infinite contexts of real life. [...] our potential to master far transfer is our competitive advantage over intelligent machines. [Hervorhebungen ergänzt]“ 
Hiermit wird einmal mehr eine strategische Perspektive (vgl. Abschn. 3.1) untermauert, da typischerweise im Strategischen Management von Wettbewerbsvorteil (,competitive advantage“) gesprochen wird. Nachfolgend liegt zwar keine wettbewerbliche, sondern eine partnerschaftliche Perspektive zugrunde, allerdings kann es auch hierfür hilfreich sein, sich seine Vorteile bewusst zu machen. Überlegungen dazu, Verbindungen zwischen weit entfernten Themen herzustellen, schließen zudem an Gedanken zum Konnektivismus als Lerntheorieströmung (vgl. Abschn. 3.2) an; ebenso wie an weitere Gedanken zu Vernetzung in den folgenden Abschnitten.

\subsection{Strategische Mensch-Maschine-Partnerschaft}

In einer Strategischen Mensch-Maschine-Partnerschaft kommt Menschen insbesondere Sinngebung zu (vgl. Kap. 1). Weitere Begriffs- und Bedeutungskategorien finden sich - wie bei Deckert, Metz und Günther (2019a) zusammengestellt - mit Kontrolle, Teilhabe, Design, Interaktion, Vernetzung und Intelligenz. Angelehnt an de Wit und Meyer (2014), die eine einzige tragfähige Definition für Strategie für illusorisch halten, wird hier für den Terminus Strategische Mensch-Maschine-Partnerschaft nicht nach einer einzigen Definition gesucht. Es werden Überlegungen zu den oben genannten Kategorien mit zugehörigen Spannungsfeldern zugrunde gelegt (vgl. Abb. 3.1).

Der Mensch setzt - bewusst oder unbewusst im Rahmen seiner Einflussmöglichkeiten - verbunden mit eigenem Denken und Handeln Schwerpunkte zu den Spannungsfeldern Strategischer Mensch-Maschine-Partnerschaft (vgl. Abb. 3.1). Diese werden aufbauend auf Deckert, Metz und Günter (2019a) vor allem mit Fokus auf nachhaltige Entwicklung wie folgt einführend charakterisiert:

\begin{tabular}{|c|c|c|}
\hline Kategorie & \multicolumn{2}{|c|}{ Spannungsfelder } \\
\hline Sinngebung & Individuell geprägte Ziele & Kollektiv geprägte Ziele \\
\hline Kontrolle & Kontrolle durch den Menschen & Kontrolle durch die Maschine \\
\hline Teilhabe & Selbstbestimmt & Fremdbestimmt \\
\hline Design & Form/Funktion & Humaner/sozialer Zweck \\
\hline Interaktion & Menschzentriert & Maschinezentriert \\
\hline Vernetzung & Fernliegendes verbinden & Naheliegendes verbinden \\
\hline Intelligenz & Menschlich & Künstlich/Technisch/Maschinell \\
\hline
\end{tabular}

Abb. 3.1 Spannungsfelder zu Strategischer Mensch-Maschine-Partnerschaft. (Quelle: https://twitter.com/Sinnmacht/status/1173102475433451520) 


\section{Sinngebung}

Ausgehend von Davenport (2016) kann Sinngebung bzw. Denken vom Großen und Ganzen her - bei Deckert und Günther (2018, o. S.) auch ,bildlich gesprochen: [...] Denken aus einer Adlerperspektive heraus" - für Strategische Mensch-Maschine-Partnerschaft als einführend verankernd gelten und ist zumindest ab einem bestimmten Niveau beim Menschen zu verorten. Der Mensch ist generell - dies sei hier angenommen - bei zunehmender Komplexität von Daten über Informationen zum Wissen und hiermit verbundener zielorientierter Steuerung (Deckert 2006, S. 37 ff. und S. 146 ff.) bezogen auf für die reale Welt typische Probleme (vgl. Kap. 1) verglichen mit Maschinen zunehmend im Vorteil. Folgende Beispiele dienen hier zu einer Verdeutlichung des Spannungsfeldes der Orientierung von Denken und Handeln an individuell und/oder kollektiv geprägten Zielen (vgl. Abb. 3.1):

\section{Beispiel für ein kollektiv geprägtes Ziel}

Ein Beispiel für ein kollektiv geprägtes Ziel ist: Strategische MenschMaschine-Partnerschaft wird genutzt, um nachhaltige Entwicklung im Großen und Ganzen zu ermöglichen bzw. zu befördern. Hier schließt im Unternehmenskontext bspw. „Sustainable Strategizing 2.0“ (Wunder 2019, S. 21 ff.) an.

\section{Beispiel für eine Verbindung von zugleich individuell und kollektiv geprägter Zielsetzung}

Strategische Mensch-Maschine-Partnerschaft kann auch auf einer Verbindung von individuell und kollektiv geprägter Zielsetzung aufsetzen beispielsweise mittels einer APP zur Unterstützung bei zielgerichteten Entscheidungen zur Ernährung, die 1) ein gesundes Leben unterstützt (individuelles Ziel) und zugleich 2) den Verzehr maßvoller Mengen beispielsweise an Fleisch fördert und somit hilft, die Welt ein Stück in Richtung nachhaltiger Entwicklung voranzubringen (kollektives Ziel).

\section{Beispiel für ein individuell geprägtes Ziel}

Ein Beispiel für ein individuell geprägtes Ziel ist (vgl. auch Kap. 1): Große gesellschaftliche Herausforderungen rund um nachhaltige Entwicklung dienen als reales Lernfeld, , anhand dessen sich Individuen auf eine zunehmend stärker technologisierte Zeit vorbereiten können, die auch geprägt ist durch [...] Digitalisierung“ (Deckert 2019a, S. 4). 
Die Ziele können eine Realisierung von Chancen und positive Zukunftsaussichten ebenso wie eine Verminderung von Risiken adressieren, wobei Risiken auch in der nachfolgenden Kategorie Kontrolle angesprochen werden.

\title{
Kontrolle
}

Algorithmen generell und ihr kommerzieller Einsatz sind mit Risiken, möglichen Nebenwirkungen und methodischen Defiziten behaftet (Brynjolfsson und McAfee 2017; Broussard 2018; WBGU 2019), denen durch eine vorausschauend gestaltete Strategische Mensch-Maschine-Partnerschaft begegnet werden sollte (Deckert 2019a). Das Spannungsfeld von Kontrolle durch Menschen und Kontrolle durch Maschinen (vgl. Abb. 3.1) muss bewusst gestaltet werden, denn vor allem auch mit Blick auf die Würde des Menschen ist von Folgendem auszugehen:

\begin{abstract}
„Der Staat kann nicht garantieren, dass sich die gesellschaftliche Dynamik daran hält, diese Unbedingtheit des Menschen stets zu berücksichtigen. Er verpflichtet sich aber mit dem ersten Satz seiner Verfassung darauf, sich selbst solchen gesellschaftlichen Dynamiken nicht anzupassen. Was das freilich im Einzelnen und in konkreten Fällen bedeutet, lässt sich nicht einfach aus dem Text des Artikels 1 GG herleiten, sondern muss Gegenstand politischer und rechtlicher Diskurse sein." (Nassehi 2019, S. 19).
\end{abstract}

Folgt man dem WBGU (2019, S. 10), so findet sich:

\begin{abstract}
„Autonome, datenbasiert selbstständig Entscheidungen treffende, technische Systeme [...] können Muster erfassen, die Menschen aufgrund der großen Datenmengen oder Komplexität verborgen bleiben. Sie können dazu beitragen, fundiertere ökonomische, politische und soziale Entscheidungen zu treffen, aber auch gesellschaftlichen Kontrollverlust, Machtmissbrauch oder die Unterminierung von Privatheit und Freiheit zur Folge haben.“
\end{abstract}

Die Ethik-Diskussion der Europäischen Kommission (2019) zum Einsatz von KI zum Wohle des Menschen ausgehend von Grundrechten und ethischen Prinzipien hat begonnen und muss konsequent fortgeführt und regelmäßig kritisch reflektiert werden.

Tipp Auf der Internetseite der Europäischen Kommission (2019) wird zu diesen Entwicklungen im Jahre 2019 unter „Ethics guidelines for trustworthy $\mathrm{Al}^{\prime \prime}$ informiert (vgl. Internetlink im Literaturverzeichnis). Seitens UN Secretary-General's High-level Panel on Digital Cooperation (2019) findet sich ebenfalls per Link im Literaturverzeichnis das Dokument "the age of digital interdependence" online verfügbar. 
Im Sinne der Nachhaltigkeit (vgl. Abschn. 2.1) fragwürdige Entwicklungen wie beispielsweise eine Verwirklichung wirtschaftlich-spekulativer Interessen mittels Digitalisierung bei nicht unwesentlichen ökologischen und/oder sozialen Folgewirkungen - sollten stets gesellschaftlichem Diskurs sowie beteiligend und mitbestimmend angelegtem Entscheidungshandeln zugänglich sein, womit eine Überleitung zur nachfolgenden Kategorie Teilhabe gegeben ist.

\section{Teilhabe}

Das Spannungsfeld von Selbstbestimmung und Fremdbestimmung (vgl. Abb. 3.1) ist hochkomplex und insbesondere sozialwirtschaftlich höchst relevant. Zunächst einmal empfiehlt es sich als Mensch zu wissen, was man gut kann und möchte (Eignung und Neigung), damit man dies im Rahmen seiner Einflussmöglichkeiten nicht ohne guten Grund an Maschinen abgibt (Deckert 2019a). Es stellen sich insbesondere Fragen nach ,voice'- und, exit'-Optionen und dazu, inwieweit im Zuge der Digitalisierung und Technisierung sozialer Dienstleistungen ein Erhalt , analoger' Infrastruktur mit der Option einer alternativen Nutzbarkeit nach Bedarf erfolgt (Deckert und Langer 2018).

Tipp Hier https://t.co/YNVCGh5fLq im Hamburger Abendblatt zeigt sich an einem Beispiel aus einer Werkstatt für Menschen mit Behinderung, dass in bestimmten Situationen Technikeinsatz Teilhabe am Arbeitsleben schaffen kann.

$\mathrm{Zu}$ Teilhabe stellt sich letztlich die Frage, wer Einfluss auf die Ausgestaltung Strategischer Mensch-Maschine-Partnerschaft hat. Hier ergibt sich die Überleitung zur Kategorie Design, denn modernes Designverständnis kann Gedanken zur ,Teilhabe der Öffentlichkeit an politischen und ökonomischen Entscheidungsprozessen“ (Schweppenhäuser 2016, S. 11 f.) einbinden.

\section{Design}

In weitem Verständnis kann Design in seiner Funktion des Lösens von Problemen (Dorst 2015) und seiner Orientierung an humanen bzw. sozialen Zwecken (Schweppenhäuser 2016) im Zuge „Großer gesellschaftlicher Herausforderungen“ (Wissenschaftsrat 2015) Potenzial für Problemlösungsbeiträge entfalten. Ein sozial geprägtes Beispiel aus einer Werkstatt für Menschen mit Behinderung wurde zu Teilhabe oben benannt. Ökologisch geprägte Beispiele können Produkte sein, die vollständig und effizient wiederverwertbar gestaltet sind. 
Tipp Für Ausführungen zu „Problem framing as a key design practice" (Dorst 2015, o. S.) kann bei Dorst (2015) unter dem im Literaturverzeichnis angegebenen Link nachgelesen werden.

Im Spannungsfeld von Form/Funktion und Humaner/sozialer Zweck (vgl. Abb. 3.1) kann Design je nach Problemstellung Positionierung erfahren. Aus dem Blickwinkel nachhaltiger Entwicklung betrachtet, darf - die Worte von Schweppenhäuser (2016, S. VIII) aufgreifend - das „Richtige und Gute“ gern zugleich auch das „Nützliche und Effiziente“ sowie das "Schöne und Attraktive“ sein, sofern sich dies für nachhaltige Entwicklung wirksam verbinden lässt.

\section{Interaktion}

An Systeme werden in der Mensch-Computer-Interaktion Anforderungen wie 1) leichte Erlernbarkeit, 2) effiziente Nutzbarkeit, 3) geringe Fehlerrate und 4) Benutzerzufriedenheit gestellt (Krömker 2009). Bei unklaren Anforderung werden systematische Prozesse wirksam, ,um benutzerfreundliche Systeme für spezifische Anwender und Anwendungskontexte zu entwickeln“ (Krömker 2009, S. 32). Im Spannungsfeld mensch- und maschinezentrierter Interaktion (vgl. Abb. 3.1) findet beides Raum, insoweit selbst dann, wenn der Mensch im Mittelpunkt steht, bis zu einem wohlüberlegten Grade erkundet und transparent gehalten werden sollte, wozu Maschinen in der Lage sind.

Tipp Zu den Empfehlungen des WBGU (2019), deren Lektüre sich empfiehlt, (vgl. Literaturverzeichnis) gehören insbesondere auch "Zulassungsstandards und "Frühwarnsysteme" für Produkte und Dienstleistungen im Bereich der Mensch-Maschine-Interaktion".

\section{Vernetzung}

Im Kontext der in Abschn. 2.2 dargestellten Entwicklungen und insbesondere in Erwartung eines „Internet of Everything (Menschen, Prozesse, Daten, Dinge) [Hervorhebung ergänzt]“ (Bauer et al. 2014, S. 17) wird klar, dass Vernetzung eine wesentliche Kategorie darstellt. Im Spannungsfeld von Naheliegendes und Fernliegendes verbinden (vgl. Abb. 3.1) besitzt nach Aoun (2017) der Mensch im Vergleich zur Maschine bei Zweiterem Vorteile. Überlegungen zu Vernetzung werden auch in Abschn. 3.2 verbunden mit Lernen und mit der Lerntheorieströmung des Konnektivismus wichtig. 
Tipp Neben Artificial Intelligence (Al) und Intelligence Augmentation (IA) findet sich der Terminus Intelligent Infrastructure (II), der mit dieser Erläuterung „[...] whereby a web of computation, data, and physical entities exists that makes human environments more supportive, interesting, and safe" (Jordan 2019, o. S.) an den Vernetzungsgedanken anschließt (vgl. Link im Literaturverzeichnis) und zur Kategorie Intelligenz überleitet.

\section{Intelligenz}

Ausgewählte Informationen dazu, wodurch menschliche Intelligenz und KI (vgl. Abb. 3.1) sich im Vergleich auszeichnen, wurden bereits in Abschn. 2.2.3 gegeben.

Tipp Hier https://www.oeaw.ac.at/detail/news/ki-wird-alle-bereichedes-lebens-umkrempeln/ können einige Gedanken zu künstlicher Intelligenz nachgelesen werden (ÖAW 2019).

Für Menschen kann in Zukunft zunehmend die Frage im Raum stehen, wofür wir uns auf unsere eigene Intelligenz bzw. auf die Intelligenz anderer Menschen verlassen sowie wofür auf KI und dies je nachdem, welche Ziele verfolgt werden (vgl. Sinngebung oben), wie Kontrolle verteilt ist bzw. werden soll (vgl. Kontrolle oben), welche Form der Teilhabe gegeben ist bzw. gegeben sein soll (vgl. Teilhabe oben), was im Design im Vordergrund stehen soll (vgl. Design oben), welche Anforderungen zur Mensch- und Maschinezentrierung gestellt werden (vgl. Interaktion oben) und welche Möglichkeiten zur Vernetzung gegeben sind bzw. sein sollen (vgl. Vernetzung oben). Dies rundet hier die Ausführungen zu Strategischer Mensch-Maschine-Partnerschaft ab.

\subsection{Lebenslanges und lebensweites Lernen}

Heute sind lebenslanges Lernen und lebensweites Lernen wichtig:

,[...] learning organizations around the world share the challenges and opportunities of the lifelong or life-wide learner - longer life spans, longer workdays, work intensification, increasing urbanization, national and transnational mobility, diversity in communities, restructured work worlds that require multiple sequential career changes, accelerating technological innovation, and global networks. [Hervorhebung ergänzt]“ (Campbell und Schwier 2014, S. 364) 
Mit lebensweitem Lernen verbindet sich, dass Lernen nicht isoliert von anderen Lebensbereichen erfolgt, sondern dass beispielsweise „Arbeiten und Lernen [...] eng verwoben gedacht und gestaltet werden können“" (Deckert 2019a, S. 23).

\section{Beispiele für lebensweites Lernen}

Im berufsbegleitenden Studium mit Praxisorientierung kann eine Verknüpfung mit für Studierende relevanten Arbeitsinhalten erfolgen. Ein weiteres konkretes Beispiel sind Teamfähigkeiten, insoweit diese im Hobbysport und zugleich im Beruf sowie in der Schule und im Studium wichtig sind. Ein drittes Beispiel ist, dass sich Studierende im Studium zwecks des Erlernens wissenschaftlicher Methoden mit relevanten sozialen Fragestellungen aus der gesellschaftlichen Praxis befassen.

Diesen Gedanken leistet die Feststellung des Stifterverbandes für die Deutsche Wissenschaft (2016) Vorschub, dass für Hochschulausbildung überfachliche Kompetenzen (71\%) wichtiger werden, wobei spezialisiertes Wissen (54\%), methodische Kompetenzen (43\%) und Grundlagenfachwissen (32\%) folgen. Auch bei O'Neil und Schutt (2014) finden sich unterstützende Überlegungen: Der sinngebende Umgang mit großen Datenmengen - beispielsweise Daten im Unternehmenskontext zu interpretieren und Bedeutung beizumessen - erfordert heute Wissen um Tools und Methoden auf akademischem Niveau, wobei „Data Scientists" sich, über Programmierung und Fertigkeiten wie in Abschn. 2.2.1 genannt hinaus, zukünftig gegebenenfalls auch durch Problemlösungsfähigkeiten verbunden mit Vielseitigkeit und Interdisziplinarität sowie Soft Skills, Stellen von guten Fragen und ethisches Herangehen auszeichnen müssen (O’Neil und Schutt 2014, S. 352 ff.).

\section{Lerntheoretische Einordnung}

Die Bewertung von Aoun (2017, S. 87) zu „far transfer“ zu Beginn von Kap. 3 kann dazu ermutigen, bewusst Verbindungen zwischen weit entfernt anmutenden Themen herzustellen. $\mathrm{Zu}$ Verbindungen (englisch: Connections) kommt eine theoretische Entwicklung in den Blick und zwar die mit Beginn des 21. Jahrhunderts aufkommende Lerntheorieströmung des Konnektivismus (englisch: Connectivism). Siemens (2006) folgend haften Behaviorismus, Kognitivismus und Konstruktivismus als Lerntheorien im 20. Jahrhundert Einschränkungen an und zwar, dass 1) Lernen stets in Personen bzw. Gehirnen stattfindet, 2) der Evaluation zum Wert von Informationen und Fähigkeiten zur Synthetisierung 
und Erkennung von Verbindungen/Mustern wenig Raum zukommt und 3) technologische Entwicklungen unbeachtet bleiben. Vor diesem Hintergrund stellt Siemens (2006) den Konnektivismus zur Diskussion, der wohl bis heute nicht überall als Lerntheorie akzeptiert ist, aber nach Campbell und Schwier (2014, S. 367) als aufkommende Lerntheorie des 21. Jahrhunderts gelten kann. Alle vier Lerntheorieströmungen können je nach Anwendungsfall Beiträge zur Beschreibung und Gestaltung von Lernen leisten. Nach Anderson und Dron (2011, S. 80 ff.) folgt die ,connectivist pedagogy“ als dritte Generation auf „cognitiv-behaviourist" und die "social constructivist [...] pedagogy“ und ist Ausgangspunkt für die nächste (also hier die 4.) Generation, die sich mit Blick auf die Befähigung von Menschen, sich mit Wissen zu verbinden und Wissensquellen zu erschließen, als (kollektiv) intelligenter erweisen könnte.

\section{Vernetzung in der Anwendung}

Für herausfordernde Problemstellungen rund um nachhaltige Entwicklung - die typischerweise interdisziplinäre Zusammenarbeit und Vernetzung erfordern kann es sich lohnen, sich folgende ausgewählte Grundgedanken zum Konnektivismus in enger Anlehnung an Siemens (2006) vor Augen zu führen und mögliche Anwendungen im eigenen Umfeld zu reflektieren (frei im Deutschen wiedergegeben):

- Lernen und Wissen finden ein Fundament in Meinungsvielfalt.

- Lernen ist ein Verknüpfungsprozess von spezialisierten Knotenpunkten oder Informationsquellen.

- Lernen kann in nicht-menschlichen Systemen erfolgen.

- Pflege und Aufrechterhaltung von Verbindungen unterstützt kontinuierliches Lernen.

- Verbindungen zwischen Feldern, Ideen und Konzepten zu sehen ist eine Kernfähigkeit.

- ...

Zur Vorbereitung auf eine stärker technologisch - und speziell digital geprägte Lebenswelt und zur Mitwirkung an der Lösung herausfordernder Problemstellungen nachhaltiger Entwicklung sind wir Menschen dazu aufgerufen, Netzwerke zu pflegen und uns zudem immer wieder neu zu vernetzen, d. h. mit Neugier und Kreativität immer neue Verbindungen zu entdecken und problemlösend zu nutzen. Neugier findet bei Willcox, Sarma und Lippel (2016, S. 9) in ihrer Bedeutung für Lernen Erwähnung verbunden mit neurowissenschaftlichen Untersuchungen und einem Hinweis auf „wonder is the beginning 
of wisdom" von Sokrates. Für zukunftsfähige Veränderung kann es Scharmer (2018b) folgend hilfreich sein, dass Menschen mit offenem Geist (Neugier), offenem Herzen (Mitgefühl) und offenem Willen (Mut) an einen Punkt kommen, von wo aus Denken und Handeln verbunden wirksam werden. Mit Neugier vernetzt Denken, Fühlen und Handeln kann ein hilfreiches Motto sein.

\section{Ausgewählte Gedanken aus dem Hochschulkontext}

Typischerweise ist ein Präsenzstudium beim Lernen in Lerngruppen, in Seminaren oder im Hörsaal in bestimmtem Maße interaktionsorientiert und Studierende können sich im Präsenzstudium nicht nur mit Informationsquellen verschiedener Art, sondern auch mit Lehrenden und unter Lernenden vernetzen. Heute bieten digitale Lernwelten neue Formen für interaktives Lerndesign, die beispielsweise auch im berufsbegleitenden Fernstudium Anwendung finden können.

Tipp Hier https://digitale-skripte.hfh-fernstudium.de/diskussionsbeitraege/html/T-19-04/T-19-04.html (abgerufen am 27.11.2019) wird Interaktives Lerndesign als Aktionsfeld und Vision am Fachbereich Technik der HFH • Hamburger Fern-Hochschule vorgestellt. Interaktion prägt das Studienkonzept der HFH von Beginn an: Das Selbststudium wird durch ein fakultatives Präsenzseminarangebot im bundesweiten Netzwerk an Studienzentren ergänzt. Heute kommt Interaktion auch digital zum Tragen.

Nach Lange und Santarius (2019, S. 24) ,sollte die Digitalisierung in den Dienst einer sozial-ökologischen Transformation gestellt werden“. Dabei ist nach Lange und Santarius (2019) Digital Literacy zentral, die Medienkompetenz, Ermächtigung zu digitaler Souveränität, digitale Nachhaltigkeitskompetenz und kritisches Denken zu übergeordneten Fragestellungen adressiert. Der Stifterverband für die Deutsche Wissenschaft (2019, S. 9) versteht unter Digital Literacy

„Grundlegende digitale Skills beherrschen, z. B. sorgsamer Umgang mit digitalen persönlichen Daten, Nutzen gängiger Software, Interagieren mit künstlicher Intelligenz"

und stellt im Rahmen von Befragungen in Unternehmen diese Rangfolge unter den folgenden sechs Kompetenzen fest: 1) Kollaboration (86\%), 2) Durchhaltevermögen $(76 \%)$, 3) Digital Literacy $(76 \%)$, 4) Unternehmerisches Handeln und Eigeninitiative (74\%), 5) Digital Learning (72\%) und 6) Agiles Arbeiten (66\%), 
wobei in Klammern jeweils angegeben ist, welcher Anteil der Mitarbeiter diese Kompetenz in fünf Jahren beherrschen soll. Vier dieser sechs Kompetenzen (1), (3), (5) und (6) zählen nach Stifterverband für die Deutsche Wissenschaft (2019) zu den Digital Citizenship Skills, die sich hier in einer Liste mit zwei - (2) und (4) - der sogenannten Classic Skills finden. Zudem werden zukünftig nach Stifterverband für die Deutsche Wissenschaft (2019) viele Personen mit Technological Skills (Technologie-Spezialisten) und hier vor allem mit Fähigkeiten zu komplexer Datenanalyse gesucht.

Tipp Unter https://www.va-bne.de/ (abgerufen am 27.11.2019) findet sich die Virtuelle Akademie Nachhaltigkeit der Universität Bremen, die digital gestütztes Lernen zu Nachhaltigkeit anbietet. Im Fachmagazin für Digitalisierung in der Lehre der Universität Hamburg mit dem Namen Synergie findet sich hier https://uhh.de/f2lec (abgerufen am 27.11.2019) die Ausgabe \#07 zum Thema Nachhaltigkeit.

Folgender Auszug zum Verständnis von Exzellenz in Lehre und Lernen findet Anschluss an eine nachhaltige Entwicklung und Digitalisierung verbindende Sichtweise und die Stärkenthese in Kap. 1.

Auszug aus "Exzellenz in Lehre und Lernen, Forschung und Transfer" (Deckert 2019b) betreffend Exzellenz in Lehre und Lernen ,[...] In Zukunft wird sich exzellentes Lehren und Lernen zunehmend auf eine stetig weiterzuentwickelnde wirkungsvolle Integration neuer Lehr-/Lernmethoden gründen. Exzellenz bedarf Lehr-/Lernstandards, die mit der Förderung individueller Stärken sowie im Lernprozess genutzter Vielfalt in der Studierendenschaft zu verbinden sind. Auf Ihre Vorteile fokussierte Präsenzlehre ist hierbei verknüpft mit - auch durch Digitalisierung geprägtem - interaktivem Lerndesign (Deckert et al. 2019b) und nach Bedarf systematisch lernprozessual verankerter Flexibilität (Deckert et al. 2018) auch für Lernende mit weiteren persönlichen und beruflichen Verpflichtungen. Lernen erfolgt handlungsorientiert auf reale Problem hin und auf Augenhöhe von (auch lernend) Lehrenden und (auch lehrend) Lernenden bei notendigen inhaltlichen Spielräumen. Es besteht Raum für Experiment, Neugier und Game-Based-Learning (Deckert et al. 2019). Hochschulisches Lernen mit neben behavioristisch, 
kognitivistisch und konstruktivistisch zusätzlich konnektivistisch geprägter Verankerung (Campbell und Schwier 2014) profitiert von Vernetzung (Menschen, Dinge) und ist Impuls für mit Arbeit verzahntes lebenslanges und -weites Lernen (Deckert 2019a) [Einige Quellen formal angepasst mit Blick auf das nachfolgende Literaturverzeichnis]. [...]“

\subsection{Vernetzung in Gesellschaft und Natur}

Die Befassung mit Vernetzung in der Gesellschaft kann vielfältige Gedanken z. B. zu Vernetzung von Menschen und Dingen (vgl. Abschn. 2.2.2), branchenspezifischer Vernetzung (vgl. beispielsweise Deckert und Saß (2020) für die Energiewirtschaft), Wertschöpfungssystemen mit vertikaler und horizontaler Vernetzung (Schuh et al. 2017), technologisch geprägter Integration (Kane et al. 2015), sozialwirtschaftlichen Wirkungs-/Handlungsfeldern (Deckert und Langer 2018) oder öffentlicher Verwaltung (Deckert 2019c) auslösen. Leinfelder (2019) fordert im Angesicht nachhaltiger Entwicklung als Herausforderung - mit Hinweis auf die Verbindung von Kultur und Natur nach A. v. Humboldt - stärkere Vernetzung in der Wissenschaft. Komplexe Probleme im Zuge nachhaltiger Entwicklung erfordern problembezogen wirksame interdisziplinäre Vernetzung.

Insoweit die Menschheit für nachhaltige Entwicklung zum Erhalt ihres planetaren Lebensraums - und damit zu ihrem eigenen Wohl - Naturgegebenheiten auf dem Planeten Erde und hier ablaufenden Prozessen Rechnung tragen muss, bietet es sich an, sich Vernetzungen in der Natur vor Augen zu führen. Vernetzung kommt zum Ausdruck in Umwandlung und Transport von Energie, die vor allem von der Sonne aus die Erde erreicht, in Bewegungen von Elementarteilchen, Ionen, Atomen und Molekülen bis hin zu makroskopischen Stoffflüssen und -kreisläufen an Land, im Wasser und in der Atmosphäre sowie disruptiven Bewegungen wie Erdrutschen oder Vulkanbrüchen, in Prozessen rund um Atmung, Ernährung, Herstellung und Nutzung von Behausung oder Entwicklung und Nutzung von Technik sowie in Kommunikation, Kooperation und Konfliktgeschehen unter Lebewesen, um hier ohne Anspruch auf Vollständigkeit ein Spektrum an Beispielen zu nennen. 
Tipp Erkenntnisse rund um das wood-wide web bieten spannende Einblicke, von denen beispielsweise einige in einem TED Talk von Suzanne Simard vorgestellt werden.

Vernetzung in der Natur auf diese Weise beispielhaft aufzuzeigen, soll hier genügen, um die sich hierin spiegelnde und das Leben prägende Vielfalt, Dynamik, Widerstandsfähigkeit und Ästhetik anzusprechen. Veränderungen an einer Stelle können unberechenbare Auswirkungen an anderer Stelle haben. Stabilität und Fragilität liegen mitunter nahe beieinander. Die aktuellen Entwicklungen lehren uns einmal mehr, dass Natur stets ein relevanter Rahmen bleibt (vgl. Abb. 3.2).

Die Natur sollte daher von der Menschheit mit Respekt und Ehrfurcht sowie mit Wertschätzung und Achtung behandelt und stets mit Weitblick und Vernunft lebenswert genutzt werden. Die Existenz von Menschen selbst zeigt, wozu Natur in der Lage ist. Ein wertschätzender Umgang mit der Natur kann mit dem wertschätzenden Umgang mit sich selber und mit seiner näheren Umgebung beginnen. Wirksame Vernetzung sorgt dafür, dass hierin stets auch ein Beitrag zum Großen und Ganzen liegt.

Tipp Es mag hilfreich sein, sich immer mal wieder zweierlei vor Augen zu führen: 1) Soziale Interaktion ist für das Lernen des Menschen von Kindesbeinen an essenziell (Meltzoff et al. 2009). 2) Für Gelingen verbunden mit großen Herausforderungen gilt: „Es geht nur gemeinsam" (Hüther 2018, S. 37).

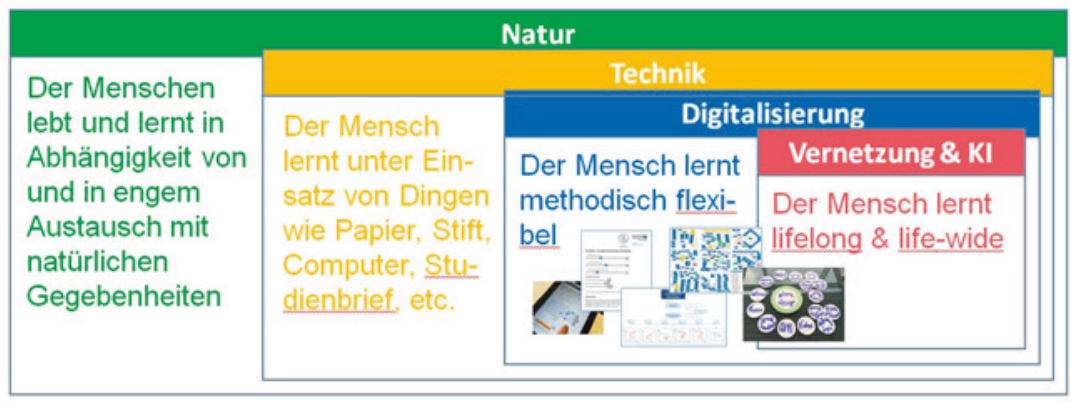

Abb. 3.2 Natur, Technik, Digitalisierung, Vernetzung und KI. (Quelle: Deckert et al. 2019b) 


\section{Aus der Coronavirus-Krise für nachhaltige Entwicklung lernen}

Die Coronavirus-Krise, die zu Beginn des Jahres 2020 weltweit Raum greift, bringt viel Leid und für das gewohnte tägliche Leben einschneidende und weitreichende Veränderungen mit sich. Diese Veränderungen tragen das Potenzial, dass Menschen in der Bevölkerung insgesamt sich intensiv mit ihren Lebensentwürfen und zugehörigen Rahmenbedingungen auseinandersetzen und dass dies wiederum in der Folge einer nachhaltigen Entwicklung zuträglich werden kann. Einige Beispiele, die insgesamt unser Denken, Fühlen und Handeln ansprechen können, sind:

- Erfahrungen zur großen Bedeutung von Solidarität im Kleinen und im Großen,

- Erfahrungen zur Bedeutung vorausschauenden Umgangs mit exponentiellen Entwicklungen,

- Wertschätzung für die Leistungen von Menschen, die in der Daseinsvorsorge arbeiten,

- Fokus darauf, was das Leben im engsten Kreis im Kern ausmachen kann, mit Blick auf Glück, Zufriedenheit und Bedürfnisse sowie mit Blick auf hiermit verbundene Herausforderungen,

- Erfahrungen mit der Arbeit im Home-Office und mit dem Einsatz digitaler Medien,

- Stärkung persönlichen Wachstums verbunden mit Bildung und Lernen,

- Reduzierung von Fernreisen und des Flugverkehrs sowie

- Erfahrungen dazu, dass stabile Verhältnisse nicht per se selbstverständlich sind und dass deren Herstellung und Erhalt jeden einzelnen immer wieder fordern können.

Die Entwicklungen in der Coronavirus-Krise machen Menschen sehr deutlich, dass das eigene Verhalten Einfluss auf die Entwicklungen im Großen und Ganzen hat. Dies erfolgt sehr nah an individuellen Lebensgewohnheiten und deren Einschränkung durch Regierungen. Die Entwicklung zum Coronavirus ist im Kleinen wie im Großen in Form von Zahlen insbesondere zu Erkrankten und dem Virus Erlegenen messbar; und dies auch bei allen Messungenauigkeiten recht eingängig. Diese Zahlen sind voraussichtliche Entwicklungen betreffend professionell mit Sachverstand zu bewerten, was uns dazu aufruft, Wissenschaft ernst zu nehmen. Auch dies macht die Coronavirus-Krise einmal mehr deutlich. Wie viele Phänomene - beispielsweise rund um den Klimawandel 
auch - ist der Coronavirus nicht direkt mit den eigenen Augen sichtbar, zugleich ist die Folgenschwere des eigenen Verhaltens aber recht unmissverständlich klar und nachvollziehbar; auch wenn dem nicht alle Mitglieder der Gesellschaft zu jedem Zeitpunkt konsequent Rechnung tragen (bspw. Nichteinhaltung eines Mindestabstands oder unvorsichtiges Verhalten verbunden mit Corona-Parties bei Jugendlichen).

Der Einsatz von Mitteln und Möglichkeiten rund um Digitalisierung ist einzuordnen in die Erfahrungen, die in einer Gesellschaft vorliegen. Hierfür ist der Einsatz von https://corona-nearby.com/ in Südkorea im Jahre 2020 ein Beispiel und dies vor dem Hintergrund von Erfahrungen dort mit dem MERS-Virus einige Jahre zuvor. Gerade da das zu Hause bleiben ein Mittel gegen die Ausbreitung eines neuen Virus sein kann, lohnt folgender Hinweis: Auch von zu Hause aus ist wirksames Denken, Fühlen und Handeln und ,persönliche Entfaltung in Gemeinschaft" (Deckert 2019a, S. 32) möglich wie das Beispiel des WirvsVirus-Hackathons der Bundesregierung im März des Jahres 2020 zeigt.

Die Coronavirus-Krise bietet die Chance dazu, zu verstehen, dass Menschen Vernetzung nicht einfach stets so gestalten können, wie es ihnen gänzlich frei beliebt, sondern letztlich nur im Einklang mit der jeweils durch die Natur vorgegebenen Vernetzung (über)leben können. Die Coronavirus-Krise trägt das Potenzial, Menschen einmal mehr die große Bedeutung von Respekt und Wertschätzung für natürliche Rahmenbedingungen vor Augen zu führen. Systeme in der Gesellschaft wie beispielsweise das Gesundheitssystem können vor dem Hintergrund exponentieller Entwicklungen wie der Verbreitung eines neuen Virus an Grenzen kommen: Wie hier http:// dipbt.bundestag.de/doc/btd/17/120/1712051.pdf auf Seite 5 ersichtlich ist, wurde die Situation von Herausforderungen, die das Gesundheitssystem nicht bewältigen kann, bereits im Jahr 2012 mittels einer damals angenommenen Coronavirus-Pandemie für Deutschland simuliert. Dieses Risiko ist also prinzipiell bekannt und auch die aktuellen Erfahrungen bieten einmal mehr die Chance, die Adäquanz der personellen und materiellen Ausstattung sowie die dahinter liegende Logistik in verschiedenen Bereichen der Gesellschaft wie dem Gesundheitssystem zielgerichtet zu hinterfragen und auszurichten. Zivilisatorische Errungenschaften wie das Gesundheitssystem sollten wir stets mit Vernunft und Weitblick erhalten und weiterentwickeln. Einmal Erreichtes ist nicht auf immer wie selbstverständlich vorhanden und wir müssen uns hierfür immer wieder persönlich einsetzen. 